\title{
Recovery of Outer Retinal Laminations on Optical Coherence Tomography After Treatment of Cancer Associated Retinopathy
}

\author{
Francisco J Irizarry ${ }^{1}$, Laura J Kopplin², Sherveen S Salek ${ }^{2}$, Grazyna Adamus², Mohamed Saleh ${ }^{2}$, Kristin \\ Biggee $^{2}$, Phoebe Lin ${ }^{2}$ and James T Rosenbaum ${ }^{2,3 *}$ \\ ${ }^{1}$ Ponce Health Science University, USA \\ ${ }^{2}$ Oregon Health \& Science University, USA \\ ${ }^{3}$ Legacy Devers Eye Institute, USA
}

Submission: February 16, 2017; Published: March 27, 2017

*Corresponding author: James T Rosenbaum, MD, Oregon Health \& Science University, Legacy Devers Eye Institute, 3375 SW Terwilliger Boulevard, Portland, OR 97239, USA, Tel: 503-464-5023; Fax 503-494-6875; Email: rosenbaj@ohsu.edu

\begin{abstract}
Purpose: To report novel optical coherence tomography findings in a case of anti- $\alpha$-enolasecancer associated retinopathy.

Observations: An elderly female presented with bilateral decreased vision and a recent diagnosis of ovarian carcinoma. Optical coherence tomography demonstrated bilateral loss of outer retinal structures and macular edema. Serum testing found antibodies against $\alpha$-enolase and 82-84kDa proteins. Outer retinal structures showed recovery, macular edema resolved and repeat anti-retinalantibody testing became negative following cancer therapy and topical difluprednate treatment.
\end{abstract}

Conclusion and importance: Cancer associated retinopathy is a paraneoplastic disease that results in damage to retinal structures through an autoimmune response. The damage is generally considered to be irreversible however, in rare cases, such as observed here, retinal structures may demonstrate recovery after treatment.

Keywords: Cancer associated retinopathy; Optical coherence tomography

\section{Introduction}

Cancer associated retinopathy (CAR) is a paraneoplastic disease in which retinal degeneration occurs as an immune response to cancer antigens sharing homology with endogenous retinal proteins [1]. Past studies have found various retinal proteins to be antigenic, which include recoverin, $\alpha$-enolase, arrestin, and transducin [2]. The inhibition of enolase, a glycolytic enzyme, results in metabolic disruption of retinal cells and the induction of apoptosis [3]. Anti- $\alpha$-enolase autoantibodies are capable of accessing tissue and targeting ganglion cells, Muller cells, and photoreceptors. It is believed that death of retinal cells is an irreversible process. We report a patient with gynecologicalCAR who experienced objective improvement in photoreceptor architecture following treatment of her underlying malignancy.

\section{Case Report}

An 80 year Hispanic female with a history of chronic, bilateral Vogt-Koyanagi-Harada associated uveitis presented to the Casey Eye Institute Uveitis Clinic for a routine follow up visit. At that time, she reported a newdiagnosis of ovarian carcinoma and had started her first chemotherapy session consisting of carboplatin and paclitaxel. Due to severe aortic stenosis, the patient was not a candidate for surgical intervention. Her vision was 20/30 bilaterally without evidence of active uveitis. Four months later she returned with a bilateral decrease in vision to $20 / 50$. The patient underwent imaging with macular volume scans centered on the fovea (Heidelberg Spectralis spectral domain ocular coherence tomography (OCT) with eye tracking software, Heidelberg, Germany) that demonstrated a disrupted inner segment/outer segment junction (ISOS) and cystoid macular edema (CME) bilaterally (Figure 1A \& 1B). Clinical and OCT findings were suspicious for CAR and anti-retinal antibody testing was pursued. The patient declined local or systemic immunosuppression specifically for her ophthalmic diseaseand continued to undergo planned chemotherapy. One month later, 
her vision had dropped to 20/500D and 20/1000S. Repeat OCT mapped to the original images continued to demonstrate loss of the ISOS junction and CME in both eyes (Figure 1C \& 1D). Serum tested for the presence of anti-retinal autoantibodies showed antibodies against $\alpha$-enolase and $82-84 \mathrm{kDa}$ proteins. Immunohistochemistry of the patient's serum showed positive staining of the photoreceptor cell layer in human retina. The patient continued to decline periocular injection or systemic immunosuppression and was prescribed difluprednate drops three times daily. Two months later, there was partial return to normal reflectivity of the IS/OS junction on OCT and the CME had improved. Six months later, there was resolved CME on OCT and the IS/OS reflectivity returned to near normal in the subfoveal region. At this time, the vision was $20 / 40$ bilaterally. The patient was instructed to stop difluprednate drops. Over the following six months, the patient's visual acuity stabilized at 20/600D and 20/500S. There was no recurrence of CME and the OCT showed normalized IS/OS reflectivity except in the fovea where there was a stable elevated outer retinal lesion OD and near-normalized ISOS reflectivity in the left macula except in the fovea (Figure 1E \& 1F). Serum was negative for anti-retinal autoantibodies on repeat testing.

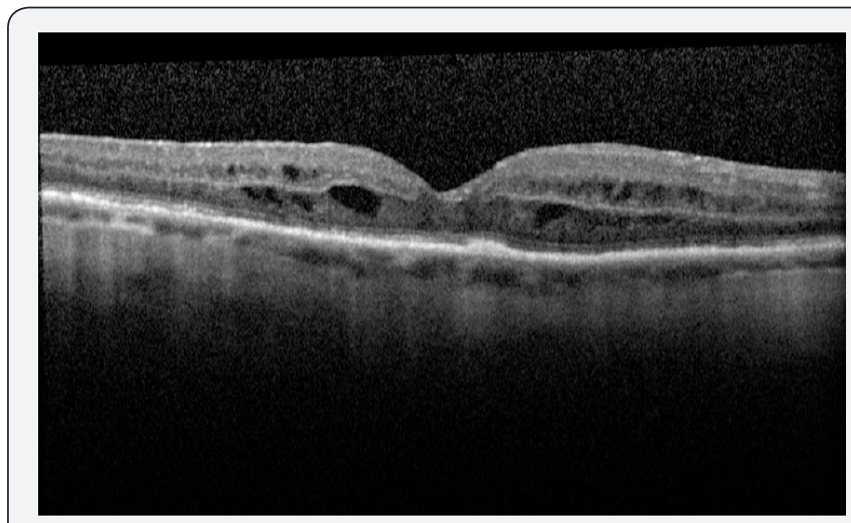

Figure 1A: OCT images of initial loss and final improvement of the ISOS junction. Loss of ISOS hyperreflectivity and concurrent CME at time of initial vision loss OD (A) and OS (B).

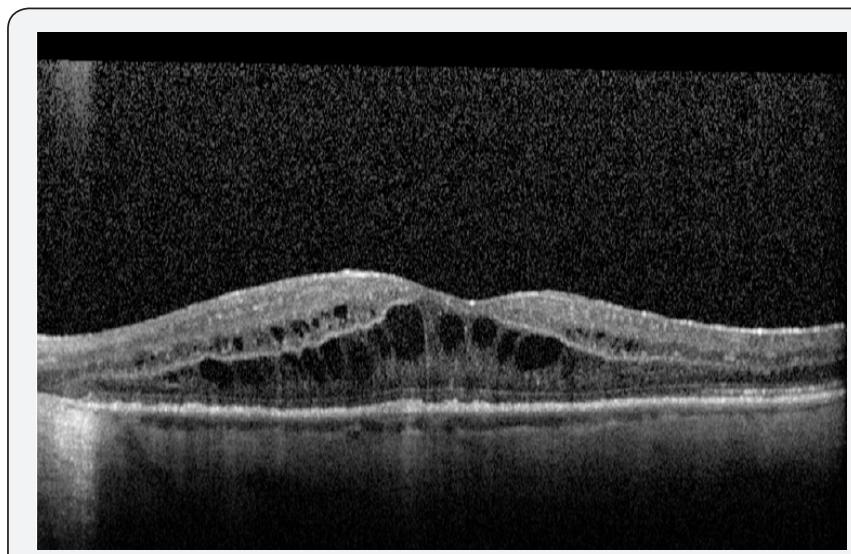

Figure 1B: OCT images of initial loss and final improvement of the ISOS junction. Loss of ISOS hyperreflectivity and concurrent CME at time of initial vision loss OD (A) and OS (B).

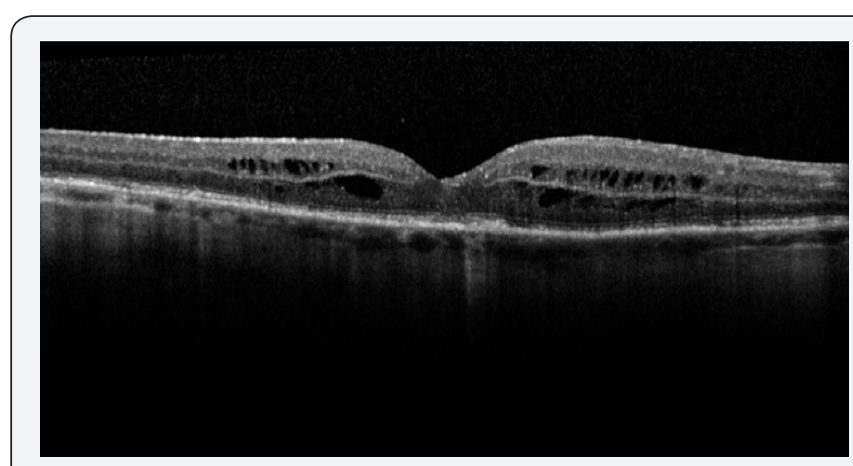

Figure 1C: Persistence of ISOS loss and CME after 1 month of observation OD (C) and OS (D).

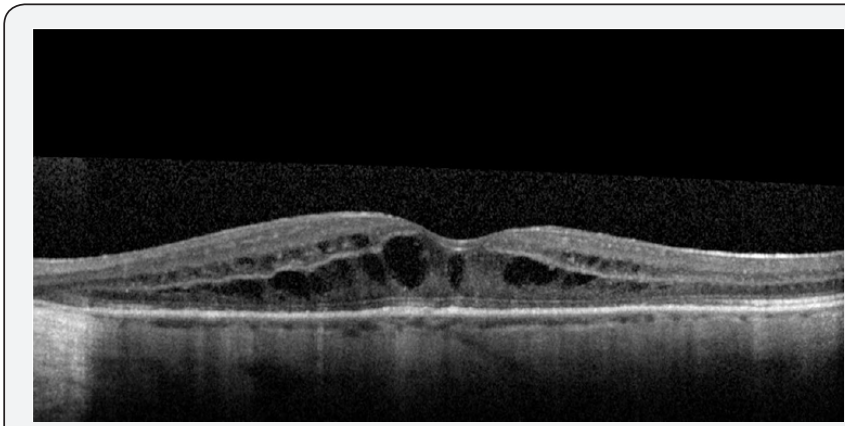

Figure 1D: Persistence of ISOS loss and CME after 1 month of observation OD (C) and OS (D).

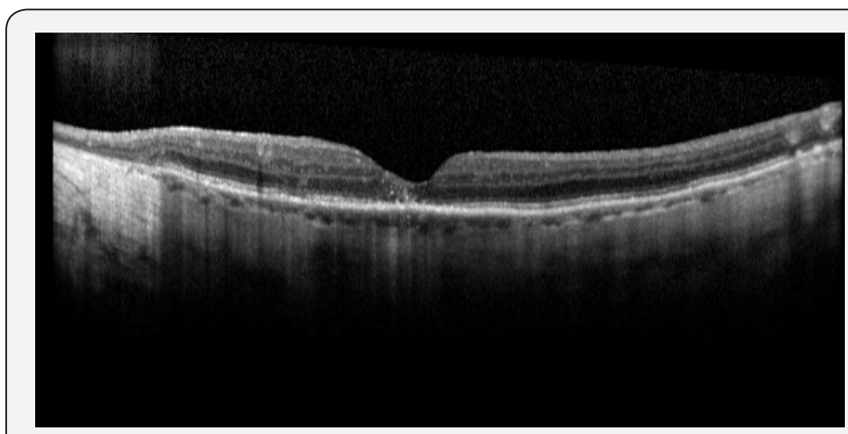

Figure 1E: Partial recovery of ISOS hyperreflectivity with residual foveal outer retinal no dularity $O D$ one year after initial findings.

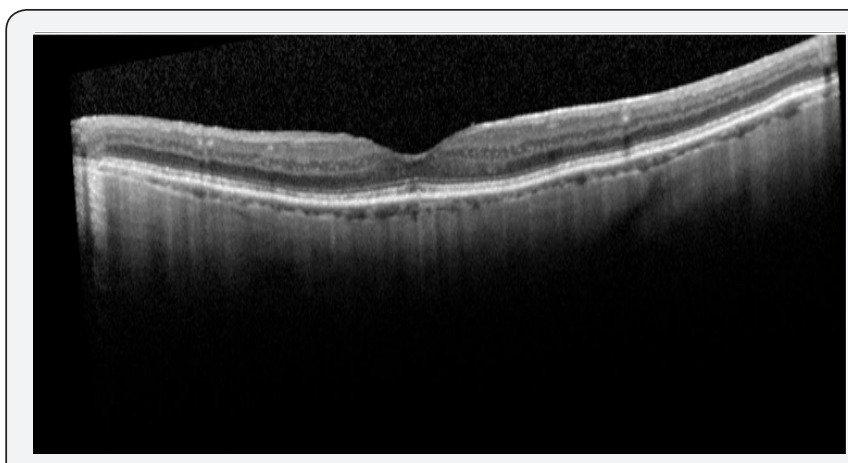

Figure 1F: Recovery of ISOS hyperreflectivity with mild residual foveal disruption one year after initial findings. OCT=optical coherence tomography, ISOS=inner segment/outer segment, $\mathrm{CME}=$ cystoid macular edema. 


\section{Discussion}

Anti-retinal autoantibodies can be detected in both retinopathy patients and healthy individuals. Individuals with gynecological CAR have a higher proportion of seropositivity than normal individuals $[4,5]$. Our patient became symptomatic after diagnosis of ovarian cancerand initiation of chemotherapy treatment. Autoantibodies may be present before the diagnosis of cancer, but it is not until they breach the blood retinal barrier that symptoms become evident [4]. Although the presence of anti-retinal autoantibodies can occur in normal patients, high antibody titers are a better indicator of retinopathy [5]. Antienolase autoantibodies affect the catalytic activity of the enzyme thus depleting glycolytic ATP, increasing levels of intracellular calcium which then induces mitochondrial-mediated apoptosis by the activation of its key elements [3].

The loss of outer retinal structures and retinal atrophy observed in autoimmune retinopathy arefrequently thought to be irreversible $[5,6]$. Partial recovery of SD-OCT outer retinal changes in a patient with CAR after treatment with rituximab has been reported, which suggests that therapy targeting B cells and consequently reducing production of anti-retinal antibodies may be beneficial [7]. Our patient showed improvement of the reflectivity of the photoreceptor IS/OS junctiondespite only local therapy with difluprednate, which was started to treat CME and reduce local inflammatory damage, but unlikely to significantly affect autoantibody production. We hypothesize that the prompt initiation of chemotherapy may have contributed to the patient's improvement by possibly decreasing tumor expression of enolase and diminishing the production of anti-retinal autoantibodies or that chemotherapeutic treatment non-specifically immunosuppressed antibody production.The recovery of outer retinal structures in the present case corresponded to antiretinal antibodies no longer being detected in the patient's serum, supporting a pathologic role for autoantibodies in our patient.Treatments that may limit the production of anti-retinal antibodies such as rituximab should continue to be studied for efficacy in CAR.

The history of prior uveitis is a potential confounder to our findings; however, the patient did not demonstrate active inflammation during this follow up period. The presence of CME may also confound the ability to image the outer retina; however, we observed patchiness of the ISOS junction outside of regions of retinal edema indicating the outer retinal changes were not a sequel of CME alone. CME is not a common manifestation of CAR, more frequently observed in non-paraneoplastic autoimmune retinopathy [8], but previous case reports of CAR-related CME suggest it is responsive to steroids, as was observed in our patient [9]. Unfortunately, the patient declined additional objective testing (visual fields, electroretinography), which would have allowed further clinical correlation.

We report a patient with CAR who experienced objective improvement in photoreceptor architecture following treatment of her underlying malignancy, a novel observation previously reported only following rituximab therapy. We also note the successful treatment of CAR-associated CME with topical difluprednate, suggesting an alternative therapy to previously reported treatments with periocular or intravitreal steroids.

\section{Conclusion}

Damage to retinal structures from CAR can be objectively captured by OCT and may demonstrate recovery after treatment in rare cases.

\section{Patient Consent}

Consent to publish the case report was not obtained. This report does not contain any personal information that could lead to the identification of the patient.

\section{Funding}

P30 EY010572 from the National Institutes of Health (Bethesda, MD), unrestricted departmental funding to the Casey Eye Institute from Research to Prevent Blindness (New York, NY).

\section{Conflict of Interest}

JTR, PL, GA. The following authors have no financial disclosures FJI, LJK, SSS, MS, KB. All authors attest that they meet the current ICMJE criteria for Authorship.

\section{References}

1. Rahimy E, Sarraf D (2013) Paraneoplastic and non-paraneoplastic retinopathy and optic neuropathy: evaluation and management. Surv Ophthalmol 58(5): 430-458.

2. Weleber RG, Watzke RC, Shults WT, Trzupek KM, Heckenlively JR, et al. (2005) Clinical and electrophysiologic characterization of paraneoplastic and autoimmune retinopathies associated with antienolase antibodies. Am J Ophthalmol 139(5): 780-794.

3. Magrys A, Anekonda T, Ren G, Adamus G (2007) The role of anti-alphaenolase autoantibodies in pathogenicity of autoimmune-mediated retinopathy. J Clin Immunol 27(2): 181-92.

4. Adamus G, Choi D, Raghunath A, Schiffman J (2013) Significance of anti-retinal autoantibodies in cancer-associated retinopathy with gynecological cancers. J Clin Exp Ophthalmol 4(6): 307.

5. Abazari A, Allam SS, Adamus G, Ghazi NG (2012) Optical coherence tomography findings in autoimmune retinopathy. Am J Ophthalmol 153(4): 750-756.

6. Pepple KL, Cusick M, Jaffe GJ, Mruthyunjaya P (2013) SD-OCT and autofluorescence characteristics of autoimmune retinopathy. Br J Ophthalmol 97(2): 139-144.

7. Or C, Collins DR, Merkur AB, Wang Y, Chan CC, et al. (2013) Intravenous rituximab for the treatment of cancer-associated retinopathy. Can J Ophthalmol 48(2): e35-e38.

8. FerreyraHA,JayasunderaT,Khan NW, HeS,LuY, etal.(2009)Management of autoimmune retinopathies with immunosuppression. Arch Ophthalmol 127(4): 390-397.

9. Moyer K, De Wilde A, Law C (2014) Cystoid macular edema from cancer-associated retinopathy. Optom Vis Sci 91(4 Suppl 1): S66-S70. 
Your next submission with Juniper Publishers will reach you the below assets

- Quality Editorial service

- Swift Peer Review

- Reprints availability

- E-prints Service

- Manuscript Podcast for convenient understanding

- Global attainment for your research

- Manuscript accessibility in different formats

(Pdf, E-pub, Full Text, Audio)

- Unceasing customer service

Track the below URL for one-step submission https://juniperpublishers.com/online-submission.php 